\title{
Expanding diagnostic tools for dysthyroid optic neuropathy: how quantitative MRI can be used to visualize and measure orbital inflammation
}

\author{
Augustin Lecler $^{1}$ (D) \\ Received: 12 May 2021 / Revised: 3 June 2021 / Accepted: 14 June 2021 / Published online: 31 July 2021 \\ (C) European Society of Radiology 2021
}

Thyroid eye disease (TED) is a chronic inflammatory disorder involving intra-orbital structures such as orbital adipose tissue and extraocular muscles [1]. Its diagnosis is based on both clinical and biological grounds $[1,2]$. Imaging might be used to confirm the diagnosis, typically showing enlarged extraocular muscles and adipose tissue infiltration of both orbits. MRI is the modality of choice to confirm the presence of subtle inflammation, which might be difficult to assess clinically, but requires urgent treatment. Corticotherapy remains the first-line treatment in patients with clinically active inflammatory TED, but other treatments, such as radiotherapy, might be useful to reduce inflammation. However, these treatments expose patients to various and potentially severe side effects [1].

In the case of active inflammation, MRI typically shows high signal intensity of the extraocular muscles and the adipose tissue as well as the lacrimal gland herniation [1, 3-7]. MRI has been reported to be more sensitive than clinical assessments in detecting orbital inflammation, identifying clinically silent inflammation of deep orbital structures, and in predicting the treatment response $[6,8,9]$. However, as there is no current gold standard for diagnosing inflammation in TED, it is impossible to ascertain that MRI is more accurate and reliable than the clinical and biological assessments.

This issue is the same for TED-related optic neuropathy $(\mathrm{ON})$, which is the most feared complication of TED and might lead to blindness [1]. Both clinical and imaging assessments have a low sensitivity and a low specificity. Progress has been made recently to improve the quality of MRI, by developing new sequences such as DIXONT2WI or IDEAL-T2WI, using the resonant frequency

Augustin Lecler

alecler@for.paris

1 Department of Neuroradiology, A. Rothschild Foundation Hospital, 25 rue Manin, 75019 Paris, France difference of water and fat protons to separate water and fat signals and applying post-processing phase correction to eliminate the effects of field inhomogeneity. These sequences show significantly fewer artifacts than conventional fat-suppressed T2WI. For example, susceptibility artifacts are frequent in the orbital region, which is surrounded by paranasal sinuses, and is therefore prone to incomplete fat-suppression at air-tissue interfaces. These sequences were reported to improve both sensitivity and specificity when evaluating inflammation in TED as compared to a conventional protocol [6].

Another promising approach is to perform a quantitative analysis of the MRI. It provides quantitative data, such as fat fractions, muscle volumes, and water T2 value for assessing extraocular muscle inflammation, or diameter and crosssectional area of the optic nerve and its sheath, water fraction of the optic nerve, and volume of the fluid in the optic nerve sheath for assessing optic neuropathy [10]. All these quantitative data might be used as biomarkers to diagnose and follow up extraocular muscle inflammation or optic neuropathy in TED. These biomarkers were reported to be reliable and specific. They were also correlated with clinical measures and other imaging measures.

Therefore, the use of quantitative data might help both radiologists and clinicians when diagnosing inflammation and/or optic neuropathy in TED. In the near future, such biomarkers might be integrated into machine learning algorithms to further improve diagnostic accuracy using MRI.

Acknowledgements Laura McMaster for editing the manuscript

Funding The authors state that this work has not received any funding.

\section{Declarations}

Guarantor The scientific guarantor of this publication is Augustin Lecler. 
Conflict of interest The author of this manuscript declares no relationships with any companies whose products or services may be related to the subject matter of the article.

Statistics and biometry No complex statistical methods were necessary for this paper.

Informed consent Not applicable

Ethical approval Not applicable

\section{Methodology}

- Editorial comment

\section{References}

1. Bahn RS (2010) Graves' ophthalmopathy. N Engl J Med 362:726738. https://doi.org/10.1056/NEJMra0905750

2. Barrio-Barrio J, Sabater AL, Bonet-Farriol E et al (2015) Graves' Ophthalmopathy: VISA versus EUGOGO classification, assessment, and management. J Ophthalmol 2015:1-16. https://doi.org/ $10.1155 / 2015 / 249125$

3. Parmar H, Ibrahim M (2008) Extrathyroidal manifestations of thyroid disease: thyroid ophthalmopathy. Neuroimaging Clin N Am 18:527-536. https://doi.org/10.1016/j.nic.2008.03.003

4. Hiwatashi A, Togao O, Yamashita K et al (2018) Diffusionweighted magnetic resonance imaging of extraocular muscles in patients with Grave's ophthalmopathy using turbo field echo with diffusion-sensitized driven-equilibrium preparation. Diagn Interv Imaging 99:457-463. https://doi.org/10.1016/j.diii.2018.02.007
5. Tortora F, Cirillo M, Ferrara M et al (2013) Disease activity in Graves' ophthalmopathy: diagnosis with orbital MR imaging and correlation with clinical score. Neuroradiol J 26:555-564. https:// doi.org/10.1177/197140091302600509

6. Ollitrault A, Charbonneau F, Herdan M-L et al (2021) Dixon-T2WI magnetic resonance imaging at 3 tesla outperforms conventional imaging for thyroid eye disease. Eur Radiol. https://doi.org/10.1007/s00330-020-07540-y

7. Gagliardo C, Radellini S, Morreale Bubella R et al (2020) Lacrimal gland herniation in Graves ophthalmopathy: a simple and useful MRI biomarker of disease activity. Eur Radiol 30:2138-2141. https://doi.org/10.1007/s00330-01906570-5

8. Tachibana S, Murakami T, Noguchi H et al (2010) Orbital magnetic resonance imaging combined with clinical activity score can improve the sensitivity of detection of disease activity and prediction of response to immunosuppressive therapy for Graves' ophthalmopathy. Endocr J 57:853-861. https://doi.org/10.1507/ endocrj.K10E-156

9. Politi LS, Godi C, Cammarata G et al (2014) Magnetic resonance imaging with diffusion-weighted imaging in the evaluation of thyroid-associated orbitopathy: getting below the tip of the iceberg. Eur Radiol 24:1118-1126. https://doi.org/10.1007/s00330-0143103-3

10. Keene KR, van Vught L, van de Velde NM et al (2021) The feasibility of quantitative MRI of extra-ocular muscles in myasthenia gravis and Graves' orbitopathy. NMR Biomed 34:e4407. https:// doi.org/10.1002/nbm.4407

Publisher's note Springer Nature remains neutral with regard to jurisdictional claims in published maps and institutional affiliations. 